\title{
Implementation of a Multimodal Multicentre Hand Hygiene Study: Evidence From Bangladesh Hospitals
}

\author{
Lutfe Ara ${ }^{1}$, Monisha Datta Trisha ${ }^{1}$, Md. Ehsanul Haque Tamal ${ }^{1}$, Noor Kutubul Alam Siddiquee ${ }^{1}$, SM Niaz Mowla ${ }^{1}$, \\ Fahima Hossain ${ }^{1}$, Tarannum Rahman ${ }^{1}$, Md. Shafiqul Alam Sarker ${ }^{1} \&$ Md. Nur Haque Alam ${ }^{1}$ \\ ${ }^{1}$ Nutrition and Clinical Services Division, International Centre for Diarrhoeal Disease Research, Bangladesh \\ (icddr,b), 68 Shaheed Tajuddin Ahmed Sarani, Mohakhali, Dhaka-1212, Bangladesh
}

Correspondence: Ms. Lutfe Ara, Head, Clinical Governance \& Systems, Nutrition and Clinical Services Division, International Centre for Diarrhoeal Disease Research, Bangladesh (icddr,b), 68 Shaheed Tajuddin Ahmed Sarani, Mohakhali, Dhaka-1212, Bangladesh. Tel: 880-173-032-0370; Office: 880-2-982-700-110. E-mail: lutfeara@icddrb.org

Received: August 8, 2019 Accepted: August 19, 2019 Online Published: September 16, 2019

doi:10.5539/gjhs.v11n11p73

URL: https://doi.org/10.5539/gjhs.v11n11p73

\begin{abstract}
Introduction: Hand hygiene ( $\mathrm{HH})$, one of the most important preventive measures of Healthcare-associated infections (HCAIs), is often neglected by healthcare workers (HCWs) in low and middle-income countries (LMICs).

Purpose: The purpose of the study is to assess the role of a multimodal intervention (MMI) for enhancing hand hygiene compliance (HHC) of HCWs in a resource-limited setting.

Methodology: A pretest-posttest quasi-experimental study was conducted in five hospitals of Bangladesh where 984 HCWs (342 physicians and 642 nurses) were selected purposively. Using a structured checklist, a direct observational assessment was carried out on HCWs' HHC both before and after the intervention. The MMI provided to HCWs comprised of: (i) system change, (ii) educational intervention, (iii) visual reminders, (iv) monitoring and performance feedback and (v) formation of infection control committees.
\end{abstract}

Results: Following intervention, overall HH compliance before and after patient contact significantly increased $(\mathrm{p}<0.00)$ to $50.1 \%$ and $57.2 \%$ respectively across all hospitals, professional categories and activities. Nurses were more compliant to $\mathrm{HH}$ than physicians $(\mathrm{OR}=1.1,95 \% \mathrm{CI}: 1.0-1.3, \mathrm{P}<0.01)$ after patient contacts. However, both groups showed equal HHC $(\mathrm{OR}=1, \mathrm{CI}$ : $0.9-1.1, \mathrm{P}=0.72)$ before patient contacts. HCWs of private hospitals were 1.5 times more compliant to HHC than that of public hospitals.

Conclusions: This study denotes that despite national policies on hand hygiene in Bangladesh, HCW's compliance to $\mathrm{HH}$ is poor. Study findings also illustrate that a multimodal $\mathrm{HH}$ program resulted in significant improvement in HCWs' HHC that deserves the potentials to assist the advancement of infection control practices targeting reduction of HCAIs.

Keywords: hand hygiene, multimodal intervention, healthcare-associated infections, low and middle- income countries

\section{Introduction}

Recognizing the merits of hand hygiene (HH), World Health Organization (WHO) accepted it as the single most effective measure to prevent healthcare associated infections (HCAIs) (Allegranzi \& Pittet, 2009) and adopted it as the core element of HCAIs reduction strategy (Pittet \& Boyce, 2001; WHO, 2009a, 2009b). The Centers for Disease Control and Prevention (CDC) and WHO published guidelines for healthcare workers (HCWs) to maintain proper HH during service delivery (Allegranzi \& Pittet, 2009; Boyce \& Pittet, 2002). Alcohol-based hand rub (ABHR) is particularly recommended in acute care as it significantly reduces HCAIs (Boyce \& Pittet, 2002; Stout, Ritchie, \& Macpherson, 2007; WHO, 2009b). Despite the delineated instructions, hand hygiene compliance (HHC) among HCWs is globally suboptimal (Allegranzi et al., 2010).

HHC is the set of behavior that ensure the adherence of HCWs to institutional HH guidelines (Aronson, 2007). Proper HH of HCWs interrupts the key pathway of pathogen transmission and reduces the incidence of HCAIs, a major public health challenge hampering the patient safety worldwide as it is responsible for increased morbidity, 
mortality and healthcare cost (Allegranzi et al., 2013; Boyce \& Pittet, 2002; WHO, 2011). Yet, on an average, $\mathrm{HHC}$ of HCWs is below $40 \%$ across the world (Erasmus et al., 2010). The rationales behind non compliance are often multi-dimensional and complex; nevertheless, knowledge, attitude and behavior of HCWs significantly affect HHC (Pittet et al., 2004; Sax, Uckay, Richet, Allegranzi, \& Pittet, 2007; Whitby, McLaws, \& Ross, 2006; Whitby et al., 2007). Education and training have had little impact on achieving long-term success in increasing HHC (Neo, Sagha-Zadeh, Vielemeyer, \& Franklin, 2016). It is not only influenced by individual perception, but also by institutional deficiency of a surveillance system. Hence insufficient resources and infrastructure as well as lack of institutional commitment and active surveillance play vital roles (Erasmus et al., 2010; Gershon et al., 1995; Pittet et al., 2008). Multi-component interventions designed to target different aspects of this major public health issue have been proven successful in establishing sustainable behavioral change regarding compliance with $\mathrm{HH}$ (Naikoba \& Hayward, 2001; Vindigni, Riley, \& Jhung, 2011). Accordingly, WHO launched a world-wide multipronged intervention program, "Clean Care is Safer Care", in 2005 to promote HH as the cornerstone of limiting HCAIs (Pittet \& Donaldson, 2005). Acknowledging the progress of the program, instead of only education and training, WHO recommends undertaking multifaceted approaches to adapt local needs in improving HHC (WHO, 2009b).

Bangladesh is a resource-limited country with higher risk of HCAIs compared to developed countries (Gurley et al., 2010). In 2011-2012, 2.3\%-10.8\% patients suffered from HCAIs in Europe (European Centre for Disease Prevention and Control, 2013) whereas, the rate is reported to be more than $30 \%$ in a tertiary hospital of Bangladesh (Hussain, Fazal, \& Ahmed, 1991). Considering the higher burden of HCAIs and associated healthcare cost in low and middle-income countries (LMICs) including Bangladesh, prevention of HCAIs is declared as a global priority (Pittet et al., 2008). To attain this goal, compliance to HH guideline is critical. In 2007, Government of Bangladesh (GoB) and WHO jointly implemented the "Clean Care is Safer Care" as a pilot program in a government hospital to increase the HHC among HCWs (WHO, 2009b). Upon outstanding success of that initiative, a national guideline on HH was established (Bangladesh Directorate General of Health Services, 2010-2011). Even after the existence of a national guideline, the most recent national survey on hand hygiene in 2014 revealed, recommended HH behavior is practiced in only $2 \%$ opportunities in Bangladesh (Ministry of Local Government et al., 2014). This poor compliance, which is a potential contributor to HCAIs, has driven us to devise context-sensitive intervention strategies in light of the existing components of WHO multimodal strategy for a sustainable culture change regarding HHC in Bangladesh. To the best of our knowledge, no study was conducted among HCWs in large-scale involving multiple types of healthcare facilities (e.g. public, private, autonomous) in Bangladesh using multimodal intervention (MMI). Therefore, this study intended to enhance HHC of HCWs in various types of healthcare facilities through a MMI. The study also aimed to assess the role of MMI in improving $\mathrm{HHC}$ and compare the HHC among different groups of HCWs as well as activities associated with risk of cross-contamination.

\section{Materials and Methods}

\subsection{Study Design}

A pre-post quasi-experimental study was carried out in three phases: (i) pre-intervention, (ii) intervention and (iii) post-intervention. In each study site, two trained research staff monitored the HH compliance through direct observation. All the observers were trained by the same instructors to ensure uniformity in observation. The observers were also responsible for monitoring, communication, and immediate feedback of HH performance to the study participants. Trainings were conducted by the principal investigator and research investigators.

\subsubsection{Pre-Intervention Phase}

Each observation period lasting for 30 minutes involved monitoring of study participants for their adherence to standard HH practices. The observational data was collected following a structured check-list. Potential HH opportunities and the actual number of episodes of HH performed by HCWs were recorded according to the predefined variables (mentioned under variables). Before commencement of the study, the participants were informed about its purpose and verbal consents were taken. Quality of data was periodically cross checked by the research investigators and the principal investigator.

\subsubsection{Intervention Phase}

A MMI was designed comprising of the following strategies:

i. System change: Availability of alcohol-based hand rub (ABHR) in sufficient quantities was ensured at all prime points of patient care with cooperation from senior management of the respective study settings.

ii. Educational intervention: A multifaceted $\mathrm{HH}$ educational intervention was provided to all participating 
HCWs, which consisted of classroom lectures with audio-visual documentaries and hands on-trainings in the wards. The educational materials included ways of pathogen transmission through hands, standard technique and duration of $\mathrm{HH}$, role of $\mathrm{HH}$ in prevention of HCAIs etc. On an average, 30 lectures were provided in each study settings and each lecture lasted for 60 minutes with discussion sessions where solutions to overcome the common constraints of standard $\mathrm{HH}$ practices and its importance were discussed (Table 1). Each participant practiced standard HH steps with practical demonstration by the trainer.

iii. Visual reminders: WHO's "How To" and "Five Moments for Hand Hygiene" (5MHH) posters were displayed at all the point of patient care in different study settings.

iv. Monitoring and performance feedback: After the intervention, compliance to standard HH practice was observed and continuous feedback was provided to the HCWs on their performances. Emphasizing the identified weaknesses, the theoretical and practical trainings were tailored accordingly and the program was reinforced.

v. Development of safety climate: To ensure continuity of HH practices, a ten member infection control committee was formed in each of the study settings. Headed by a physician in charge, the committees consisted of Medical Officers, Staff Nurses, Residential Medical Officer and administrative personnel. The members of the committee were selected from the participants who received the training on $\mathrm{HH}$ practices. The committee was responsible for reporting HH practices among HCWs, ensuring supply of $\mathrm{HH}$ materials, sustaining the program and bringing system and cultural changes.

\subsubsection{Post-Intervention Phase}

After completion of the intervention phase, the participants were further observed for their rates of compliance to the standard $\mathrm{HH}$ practices. The compliance was measured in the same procedure as followed in the pre-intervention stage.

\subsection{Setting and Participants}

The study was conducted in three different periods: November 2012- January 2014, July 2014- September 2015, February 2016- April 2017 at five different hospitals of Mymensingh, Dhaka, Sirajganj and Sunamganj districts. The description of each facility is given in Table 1 . To maintain the anonymity and confidentiality, names of the hospitals were coded. The hospitals were selected purposively on the basis of study objectives, absence of any active program addressing $\mathrm{HH}$ and facility's own interest to participate in the study. Total 984 participants including 342 physicians and 642 nurses were selected by purposive sampling. All physicians and nurses, involved in direct patient care with the hospitalized patients during the study period, were invited and those who agreed to take part were included in the study.

Table 1. Characteristics of the study sites

\begin{tabular}{llllllll}
\hline \multirow{2}{*}{ Facilities } & \multirow{2}{*}{ Location } & \multirow{2}{*}{ Facility type } & Study Period & \multicolumn{2}{c}{ Number of physicians } & \multicolumn{2}{c}{ Number of nurses } \\
\cline { 7 - 8 } & & & $2012-13$ & 181 & 120 & 97 & 96 \\
\hline HosA & Mymensingh & Private, tertiary & $2014-15$ & 217 & 161 & 149 & 136 \\
HosB & Dhaka & Autonomous, tertiary & Participated & Total & Participated \\
HosC & Sirajganj & Government, secondary & $2014-15$ & 59 & 41 & 271 & 262 \\
HosD & Sunamganj & Government, secondary & $2016-17$ & 22 & 14 & 134 & 134 \\
HosE & Sunamganj & Private, secondary & $2016-17$ & 6 & 6 & 14 & 14 \\
\hline Total & & & & 485 & 342 & 665 & 642 \\
\hline
\end{tabular}

Note. Hos $\mathrm{A}=$ Hospital $\mathrm{A}, \operatorname{Hos} \mathrm{B}=$ Hospital $\mathrm{B}, \operatorname{Hos} \mathrm{C}=$ Hospital $\mathrm{C}, \operatorname{Hos} \mathrm{D}=$ Hospital $\mathrm{D}$, HosE $=$ Hospital $\mathrm{E}$.

\subsection{Variables}

The outcome variables were: (i) HHC before patient contact- "Disinfection of hands with ABHR before each patient care activities", (ii) HHC after patient contact- "Disinfection of hands with ABHR after each patient care activities."

HHC to patient care activities based on risk of cross-contamination was also measured and was categorized as 
follows: (i) Low risk activities: Touching sterile goods, making clean bed, making contact with patient notes, telephone and computer and administering oral and topical medicines during medication rounds. (ii) Medium risk activities: Stripping a non-soiled bed, making patient contact such as hand shake, operating medical devices in immediate patient environment, helping to move patient in/out of bed, cleaning beds and/or furniture, observing vital signs, setting up and removing intravenus fluids donning and removing gloves, performing bed bath and washing patients. (iii) High risk activities: Dealing with bodily secretions (urine, feces, blood) e.g. catheter bags, suctioning, tracheostomy care, wound dressings, phlebotomy, Cannulation and giving injections.

\subsection{Data Analysis}

Data collected from each phase of the study were statistically analyzed using Stata 13 . HHC was determined as the proportion of actual hand rubbing episodes and potential hand rubbing opportunities. Chi-square test was performed to compare the change in HHC between pre and post intervention period as the outcomes of interest are categorical variables. Increase in HHC was calculated at $95 \%$ confidence interval and $\mathrm{P}$ value of $<0.05$ was considered statistically significant. To assess the strength of association between binary outcome variables, Binomial logistic regression model was fitted with the period of observation (pre vs- post intervention), professional category (nurses vs- physicians), and type of healthcare facilities (public, private and autonomous) as independent variables.

\subsection{Ethical Consideration}

Institional Review Board (IRB) of icddr,b approved this study and the IRB approval number is PR\#17127. Informing the aims and objectives of the study, written permission was taken from the administrative authority of each study hospital through a Memorandum of Understanding (MoU). The participants were also provided with the information about the purpose of the study as well as risks and benefits associated with voluntary participation in it. Thus informed verbal consents were obtained from all the participants prior enrollment. The confidentiality of the collected data was ensured and the access kept limited to the Principle Investigator (PI) only. No personally identifiable information of the study participants was recorded throughout the study.

\section{Results}

Total 9,259 HH opportunities were observed and recorded across the five study hospitals: 4685 in pre-intervention phase and 4574 in post-intervention phase. The HHC of HCWs in five study hospitals, prior and post intervention is given in Table 2 .

Table 2. Increase in hand hygiene compliance after intervention

\begin{tabular}{|c|c|c|c|c|}
\hline \multirow[b]{2}{*}{ Variables } & \multicolumn{2}{|c|}{ Hand hygiene compliance (\%) } & \multirow{2}{*}{$\begin{array}{l}\text { Increase in } \mathrm{HHC} \text { from pre-intervention } \\
\text { period to post-intervention period }(\%)\end{array}$} & \multirow[b]{2}{*}{$P$ value } \\
\hline & $\begin{array}{l}\text { Pre-intervention } \\
\text { Period }\end{array}$ & $\begin{array}{l}\text { Post-intervention } \\
\text { Period }\end{array}$ & & \\
\hline \multicolumn{5}{|c|}{ Before patient contact } \\
\hline HosA & $15(9.1 \%)$ & $83(50.3 \%)$ & $41.2 \%$ & 0.000 \\
\hline HosB & $8(2.8 \%)$ & $140(51.8 \%)$ & $49.0 \%$ & 0.000 \\
\hline $\mathrm{Hos} \mathrm{C}$ & $7(2.7 \%)$ & $131(55.3 \%)$ & $52.6 \%$ & 0.000 \\
\hline HosD & $1(0.04 \%)$ & $1148(44.7 \%)$ & $44.7 \%$ & 0.000 \\
\hline HosE & $25(1.9 \%)$ & $779(59.5 \%)$ & $57.6 \%$ & 0.000 \\
\hline Overall & $56(1.2 \%)$ & $2281(50.2 \%)$ & $49.0 \%$ & 0.000 \\
\hline \multicolumn{5}{|c|}{ After patient contact } \\
\hline HosA & $19(11.5 \%)$ & $95(57.6 \%)$ & $46.1 \%$ & 0.000 \\
\hline HosB & $20(7.0 \%)$ & $159(60.4 \%)$ & $53.4 \%$ & 0.000 \\
\hline $\mathrm{Hos} \mathrm{C}$ & $11(4.2 \%)$ & $142(58.2 \%)$ & $54.0 \%$ & 0.000 \\
\hline HosD & $8(0.3 \%)$ & $1415(55.0 \%)$ & $54.7 \%$ & 0.000 \\
\hline HosE & $45(3.4 \%)$ & $827(63.2 \%)$ & $59.8 \%$ & 0.000 \\
\hline Overall & $103(2.2 \%)$ & $2638(57.9 \%)$ & $55.7 \%$ & 0.000 \\
\hline
\end{tabular}

Note. $\mathrm{HHC}=$ Hand Hygiene Compliance. 
Overall HHC before patient contact at the five study settings significantly improved $(\mathrm{P}<0.00)$ from $1.2 \%$ to $50.1 \%$ in post-intervention phase. Remarkable improvement $(\mathrm{P}<0.00)$ in overall HHC was also observed after patient contact from $2.2 \%$ to $57.2 \%$ after the MMI. The rate of $\mathrm{HHC}$ after the MMI varied among the facilities. After the intervention, the overall $\mathrm{HHC}$ rates before patient contact in public, autonomous and private hospitals were $45.4 \%, 52.8 \%$ and $58.5 \%$ respectively, while the same after patient contact were $54.7 \%, 59.9 \%$ and $61.3 \%$ respectively (Figure 1).

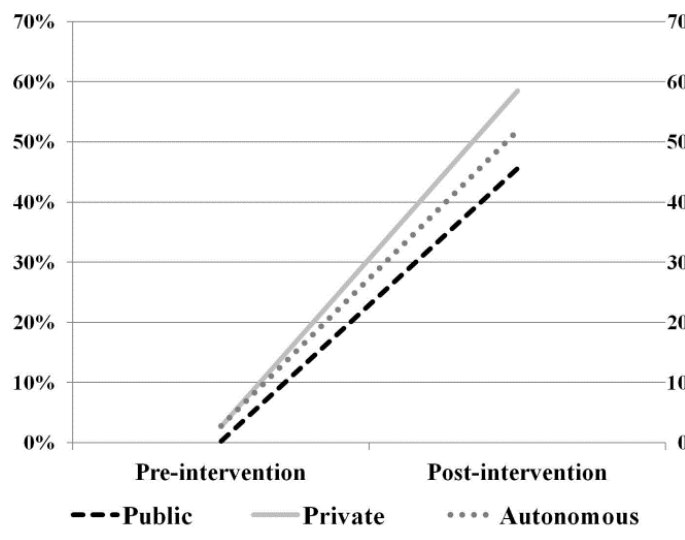

(a) HHC before patient contacts

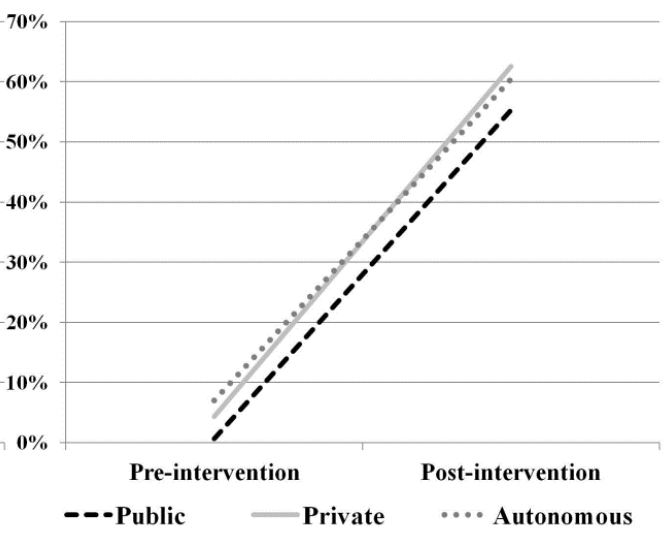

(b) HHC after patient contacts

Figure 1. Comparison of HHC by type of facilities before and after patient contacts

The results of logistic regression to consider the transform in HHC in five study hospitals over the whole study period are presented in Table 3. Substantial improvements in post intervention period both before $(\mathrm{OR}=83.1,95 \%$ CI: $63.4-108.8, \mathrm{P}<0.01)$ and after $(\mathrm{OR}=59.4,95 \% \mathrm{CI}: 48.5-72.9, \mathrm{P}<0.01)$ contacting patients have been achieved. This outstanding advancement was due to the very low or almost no compliance at the pre-intervention phase. Nurses showed higher compliance $(\mathrm{OR}=1.1,95 \% \mathrm{CI}: 1.0-1.3, \mathrm{P}<0.01)$ than that of physicians after patient contacts but both the groups were equally compliant $(\mathrm{OR}=1.0, \mathrm{CI}: 0.9-1.1, \mathrm{P}=0.72)$ before patient contacts. Overall, private facilities showed significantly greater compliance (before patient contacts: 1.5 times and after patient contacts: 1.3 times) than the public facilities.

Table 3. Odds Ratio (with 95\% CI) for Hand Hygiene Compliance from binary logistic regression

\begin{tabular}{llllll}
\hline & HH Before Patient Contact & HH After Patient Contact & Low Risk & Medium Risk & High Risk \\
\hline Phase & & & & & \\
Pre Intervention & Reference & Reference & Reference & Reference & Reference \\
& $83.2^{* *}$ & $61.3^{* *}$ & $108.6^{* *}$ & $42.8^{* *}$ & $47.2^{* *}$ \\
Post Intervention & $(63.5,109.0)$ & $(50.0,75.1)$ & $(67.0,176.0)$ & $(32.9,55.8)$ & $(36.8,60.5)$ \\
\hline Level of Hospital & & & & & \\
Secondary & Reference & Reference & Reference & Reference & Reference \\
Tertiary & 1.15 & $1.2^{*}$ & $0.7^{* *}$ & 0.9 & $0.6^{* *}$ \\
& $(0.9,1.3)$ & $(1.0,1.4)$ & $(0.6,0.8)$ & $(0.8,1.1)$ & $(0.5,0.8)$ \\
\hline Type of Hospital & & & & & \\
Public & Reference & Reference & Reference & Reference & Reference \\
Private & $1.5^{* *}$ & $1.3^{* *}$ & 1.2 & $1.8^{* *}$ & 1 \\
& $(1.3,1.7)$ & $(1.2,1.5)$ & $(1.0,1.3)$ & $(1.6,2.1)$ & $(0.8,1.2)$ \\
Autonomous & $1.3^{*}$ & $1.3^{* *}$ & 1 & $1.7^{* *}$ & 1 \\
& $(1.0,1.5)$ & $(1.1,1.6)$ & $(0.8,1.2)$ & $(1.3,2.1)$ & $(0.8,1.4)$ \\
\hline
\end{tabular}




\begin{tabular}{lll}
\hline \multicolumn{2}{l}{ Type of healthcare workers } \\
Nurse & Reference & Reference \\
& 1 & $0.8^{* *}$ \\
Physician & $(0.9,1.1)$ & $(0.7,0.9)$
\end{tabular}

Note. $*=\mathrm{P}<0.05, * *=\mathrm{P}<0.01, \mathrm{HH}=$ Hand Hygiene, $\mathrm{CI}=$ Confidence Interval.

Compared to public healthcare facilities, private facilities showed better compliance both in low risk $(\mathrm{OR}=1.2$, 95\% CI: 1.0-1.3, $\mathrm{P}=0.07)$ and medium risk activities $(\mathrm{OR}=1.8,95 \% \mathrm{CI}: 1.6-2.1, \mathrm{P}<0.01)$. However, in case of high risk activities, rate of compliance did not vary significantly among the type of hospitals. But the striking fact was rate of compliance in activities associated with all type of risks of contamination were found to be substantially greater in the post intervention phase than those in pre-intervention, which is obvious as these activities were rarely practiced before providing intervention. HHC in activities associated with risk (low, medium and high) of transmission of infection is compared in Figure 2.

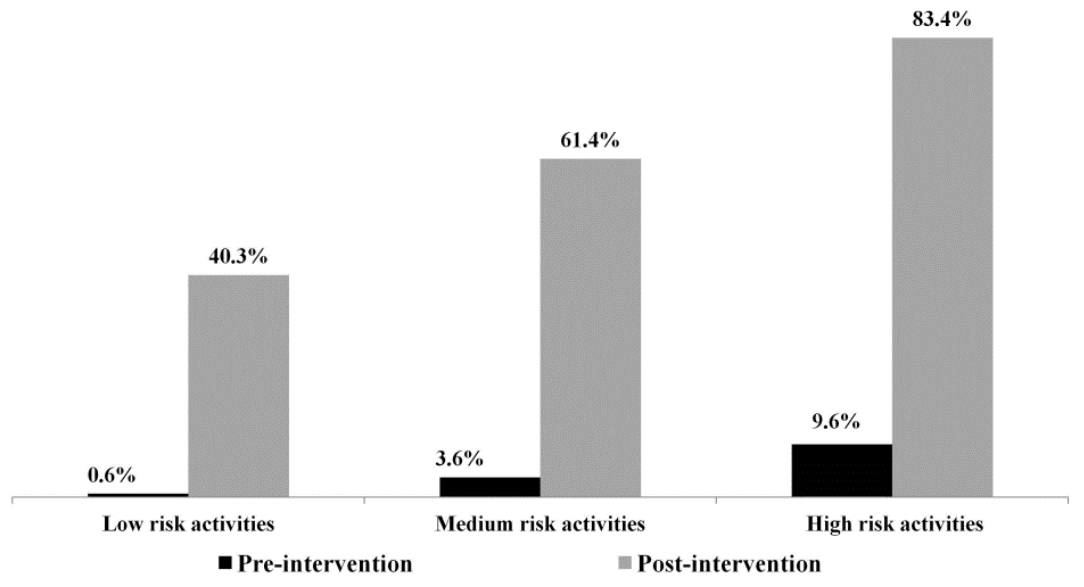

Figure 2. HHC rate of HCWs in activities associated with risk for cross-transmission

\section{Discussion}

Since HHC is influenced by multiple predictors, the study aimed to improve the HHC of HCWs through a multi-dimensional approach as well as to assess the role of MMI in improvement. This multimodal intervention study substantially improved the HHC of HCWs in all study settings, but improvement was more apparent in private facilities. Improvements were also more marked for high-risk activities, after patient contact, prominently among nurses.

At baseline, the overall HHC before and after patient contact as observed in this study was below $10 \%$ which was unexpectedly low. However, this result is concordant with the findings of a previous estimate of $6 \%$ use of alcohol sanitizer among HCWs in Bangladesh (Ministry of Local Government et al., 2014). Following the MMI, the average use of ABHR remarkably increased to $49 \%$ and $55 \%$ before and after patient contact respectively. These HHC rates are lower than a similar intervention study in Rwanda where HHC was 68.9\% (Holmen et al., 2016). In addition to education and reminders, the former study provided ABHR bottles for HCWs, so the HHC was notably higher in that study. However, the observed HHC rates of this study are higher than $44.07 \%$ hand rub use in Nigeria (Uneke et al., 2014). The difference in the findings might be related to the differences of participants in two settings. The Nigerian study assessed the HHC in all group of HCWs i.e. nurses, physicians, midwives, therapists etc.; so the overall $\mathrm{HHC}$ was slightly lower.

Higher compliance was marked after patient contact than that of before patient contact. It matches the pattern frequently revealed by other researchers (Allegranzi et al., 2013; Barahona-Guzmán et al., 2014; Horng et al., 2016; Mahfouz, Al-Zaydani, Abdelaziz, El-Gamal, \& Assiri, 2014; Mu et al., 2016; Salama, Jamal, Al Mousa, Al-AbdulGhani, \& Rotimi, 2013; Santosaningsih et al., 2017; Uneke et al., 2014). The fear of acquiring infection through patient contact might motivate the HCWs to clean their hands more after touching patients in order to protect themselves (Allegranzi et al., 2013; Randle, Arthur, \& Vaughan, 2010). Perceived risks associated with 
cross-contamination might also be the cause of higher compliance found during high risk activities. This finding corroborates well with earlier studies (Amazian et al., 2006; Lam, Lee, \& Lau, 2004; Pittet et al., 2004).

In post intervention phase, standard $\mathrm{HH}$ was practiced equally by physicians and nurses before patient contact; but nurses were more likely to clean hands than physicians after patient contact. In general, the adherence was greater among nurses than physicians, which is consistent with preceding studies (Horng et al., 2016; Lam et al., 2004; Mahfouz et al., 2014; Nteli et al., 2014; Randle, Clarke, \& Storr, 2006). This might be due to the fact that nurses more frequently come to the close contact of patients than physicians (Lam et al., 2004). In Bangladesh, general procedures (e.g. cannulation, catheterization etc.) are performed commonly by nurses (Asare, Enweronu-Laryea, $\&$ Newman, 2009), so we assume the level of awareness among nurses is greater than physicians. Physicians are probably more involved with diagnosis and treatment and less concerned about prevention. As noted by previous studies, high workload might also be associated with non-adherence of physicians (Erasmus et al., 2010; Pittet et al., 2004).

Although there was overall improvement in HHC upon implication of the MMI, considerable variance was observed in unit-level. Each hospitals included in this study were unique in their type, infrastructure, care deliveries, patient load and economics. Comparably, private hospitals showed highest adherence than autonomous and public hospitals. This finding is in agreement with another study (Rosenthal, McCormick, Guzman, Villamayor, \& Orellano, 2003) in which the author inferred that, better administrative support and accountability in private facilities persuaded the HCWs to act according to the HH guidelines. Among the five study hospitals, hospital $\mathrm{E}$ achieved the highest post-intervention compliance rates. This was a private facility with the lowest capacity and least number of HCWs, which might had some role on the elevated HHC rate of this facility. Besides, the concern of the private facilities for their reputation might also drive them for further improvement.

The study did not meticulously explore the possible influences on HH behavior of HCWs but lack of essential HH infrastructure and infection control management, overcrowding of patients as well as poor supply of $\mathrm{HH}$ materials were found as barriers to absolute HHC, which is similar to former studies (Horng et al., 2016; Lee et al., 2011; Pittet \& Boyce, 2001; Shahida et al., 2016; Whitby et al., 2006). At healthcare facilities in Bangladesh, sanitizers were reported to be available at only 32-39\% locations for physicians and 39-51\% for nurses (Ministry of Local Government et al., 2014). Autonomous and private facilities have more supplies of ABHR than government facilities, but maintenance of supplied products is poor in all sectors (Ministry of Local Government et al., 2014; Rosenthal et al., 2003). However, supply does not always ensure availability. From our observation, ABHR was often not available at the point of care at all facilities in spite of sufficient supply. Regardless of intense intervention, hospital authorities were not adequately motivated to boost ABHR availability in few cases. It suggests that, the health benefits of standard $\mathrm{HH}$ practice may be inexplicit to hospital administrative authorities. Yet, the intervention dynamically stimulated the administrative body to remove some system constraints and establish regular monitoring of $\mathrm{HH}$ practice through an infection control committee.

Absence of a comparator group is one of the limitations of this study. As the intervention was multimodal, it is also impossible to assess the relative efficacy of each component. However, in a meta-analysis of $\mathrm{HH}$ care bundles, bundles with education, feedback, reminder, access to ABHR and administrative support were found to be associated with considerable improvement in HH adherence (Schweizer et al., 2013). Though direct observation is the gold standard for measuring HHC, it possesses the inherent weakness of Hawthorne effect (Chhapola \& Brar, 2015). To minimize this effect, the participants were clearly informed about the purpose of the study but they were kept unaware of the exact date and time of observations. The study was unique by its multi-component and multicentre nature. From that point of view, this study is the first of its kind in Bangladesh. To render a general depiction of the actual scenario of the country, the study hospitals were selected from four distinct districts located at three different geographical regions of Bangladesh. All type of facilities - public, private and autonomous was included to induce generalizability. Furthermore, the study assessed the HHC in different patient care activities based on risks associated with cross-contamination, which was not determined formerly by any study in Bangladesh.

\section{Conclusion}

This multicenter study generates important evidence for enhancing HHC through effective and low cost MMI in resource poor settings like Bangladesh. Still, further investigations are required to identify and overcome the barriers to absolute compliance. In addition to the present MMI, we recommend increased supply of ABHR, as it is a more practical solution for overcrowded settings especially with poor hand washing facilities. Above all, being an LMIC, Bangladesh should concentrate on system change instigating the culture of accountability at both individual and institutional level for sustained compliance. Without long-standing institutional commitment for 
$\mathrm{HH}$ promotion, retaining progress is ambitious.

\section{Acknowledgements}

The authors thank the participating members of the medical and nursing staffs for their cooperation and participation in this study. They are particularly indebted to the Head of Hospital Administration of the study sites for their support. Authors of this article express their deepest gratitude to GSK for funding this research initiative. The funding body has no involvement in study design, data collection, analysis, interpretation, writing report, and submission of the article for publication. They are also grateful to Prof. John Clemens MD, the Executive Director of icddr,b and Dr. Tahmeed Ahmed, Senior Director of icddr,b, for their support in implementation of this study. icddr,b is thankful to the Government of Bangladesh, Canada, Sweden and the UK for providing core/unrestricted support.

\section{Data Statement}

Research data are restricted to the authors who have permission from Research Administration of icddr,b and thus not publicly available. For data access and further information, request can be sent to corresponding author.

\section{Competing Interests Statement}

The authors declare that there are no competing or potential conflicts of interest.

\section{References}

Allegranzi, B., Gayet-Ageron, A., Damani, N., Bengaly, L., McLaws, M. L., Moro, M. L., . . Storr, J. (2013). Global implementation of WHO's multimodal strategy for improvement of hand hygiene: A quasi-experimental study. The Lancet infectious diseases, 13(10), 843-851. https://doi.org/10.1016/S1473-3099(13)70163-4

Allegranzi, B., \& Pittet, D. (2009). Role of hand hygiene in healthcare-associated infection prevention. Journal of Hospital Infection, 73(4), 305-315. https://doi.org/10.1016/j.jhin.2009.04.019

Allegranzi, B., Sax, H., Bengaly, L., Riebet, H., Minta, D. K., Chraiti, M. N., . . Pittet, D. (2010). Successful implementation of the World Health Organization hand hygiene improvement strategy in a referral hospital in Mali, Africa. Infection Control \& Hospital Epidemiology, 31(2), 133-141. https://doi.org/10.1086/649796

Amazian, K., Abdelmoumene, T., Sekkat, S., Terzaki, S., Njah, M., Dhidah, L., . . Fabry, J. (2006). Multicentre study on hand hygiene facilities and practice in the Mediterranean area: results from the NosoMed Network. Journal of Hospital Infection, 62(3), 311-318. https://doi.org/10.1016/j.jhin.2005.09.023

Aronson, J. K. (2007). Compliance, concordance, adherence. Br J Clin Pharmacol, 63(4), 383-384. https://doi.org/10.1111/j.1365-2125.2007.02893.x

Asare, A., Enweronu-Laryea, C. C., \& Newman, M. J. (2009). Hand hygiene practices in a neonatal intensive care unit in Ghana. The Journal of Infection in Developing Countries, 3(05), 352-356. Retrieved from: https://www.ncbi.nlm.nih.gov/pubmed/19759504

Bangladesh Directorate General of Health Services. (2010-2011). National Guideline On Hand Hygiene For Prevention of Hospital Acquired Infection (HAI). Retrieved April 03, 2019, from http://hsmdghs-bd.org/Documents/Doc_NATIONAL\%20GUIDELINE\%20ON\%20HAND\%20HYGIENE. pdf

Barahona-Guzmán, N., Rodríguez-Calderón, M. E., Rosenthal, V. D., Olarte, N., Villamil-Gómez, W., Rojas, C., . . Menco, A. (2014). Impact of the International Nosocomial Infection Control Consortium (INICC) multidimensional hand hygiene approach in three cities of Colombia. International Journal of Infectious Diseases, 19, 67-73. https://doi.org/10.1016/j.ijid.2013.10.021

Boyce, J. M., \& Pittet, D. (2002). Guideline for hand hygiene in health-care settings: recommendations of the Healthcare Infection Control Practices Advisory Committee and the HICPAC/SHEA/APIC/IDSA Hand Hygiene Task Force. American journal of infection control, 30(8), S1-S46. Retrieved from https://www.ncbi.nlm.nih.gov/pubmed/12418624

Chhapola, V., \& Brar, R. (2015). Impact of an educational intervention on hand hygiene compliance and infection rate in a developing country neonatal intensive care unit. International journal of nursing practice, 21(5), 486-492. https://doi.org/10.1111/ijn.12283

Erasmus, V., Daha, T. J., Brug, H., Richardus, J. H., Behrendt, M. D., Vos, M. C., \& van Beeck, E. F. (2010). Systematic review of studies on compliance with hand hygiene guidelines in hospital care. Infection Control 
\& Hospital Epidemiology, 31(3), 283-294. https://doi.org/10.1086/650451

European Centre for Disease Prevention and Control. (2013). Point prevalence survey of healthcare-associated infections and antimicrobial use in European acute care hospitals. Stockholm: ECDC. Retrieved from https://ecdc.europa.eu/sites/portal/files/media/en/publications/Publications/healthcare-associated-infectionsantimicrobial-use-PPS.pdf

Gershon, R. R., Vlahov, D., Felknor, S. A., Vesley, D., Johnson, P. C., Delcios, G. L., \& Murphy, L. R. (1995). Compliance with universal precautions among health care workers at three regional hospitals. American journal of infection control, 23(4), 225-236. https://doi.org/10.1016/0196-6553(95)90067-5

Gurley, E. S., Zaman, R. U., Sultana, R., Bell, M., Fry, A. M., Srinivasan, A., . . Luby, S. P. (2010). Rates of hospital-acquired respiratory illness in Bangladeshi tertiary care hospitals: results from a low-cost pilot surveillance strategy. Clinical infectious diseases, 50(8), 1084-1090. https://doi.org/10.1086/651265

Holmen, I. C., Seneza, C., Nyiranzayisaba, B., Nyiringabo, V., Bienfait, M., \& Safdar, N. (2016). Improving hand hygiene practices in a rural hospital in sub-Saharan Africa. Infection control \& hospital epidemiology, 37(7), 834-839. https://doi.org/10.1017/ice.2016.71

Horng, L. M., Unicomb, L., Alam, M. U., Halder, A. K., Shoab, A. K., Ghosh, P. K., . . Luby, S. P. (2016). Healthcare worker and family caregiver hand hygiene in Bangladeshi healthcare facilities: results from the Bangladesh National Hygiene Baseline Survey. Journal of Hospital Infection, 94(3), 286-294. https://doi.org/10.1016/j.jhin.2016.08.016

Hussain, T., Fazal, M., \& Ahmed, A. (1991). Nosocomial infection-A cross-sectional study in the surgical wards of Dhaka Medical College Hospital. J. of Preventive and Social Med, 10(2), 10-13.

Lam, B. C., Lee, J., \& Lau, Y. L. (2004). Hand hygiene practices in a neonatal intensive care unit: a multimodal intervention and impact on nosocomial infection. Pediatrics, 114(5), e565-e571. https://doi.org/10.1542/peds.2004-1107

Lee, A., Chalfine, A., Daikos, G. L., Garilli, S., Jovanovic, B., Lemmen, S., . . Rubinovitch, B. (2011). Hand hygiene practices and adherence determinants in surgical wards across Europe and Israel: a multicenter observational study. American journal of infection control, 39(6), 517-520. https://doi.org/10.1016/j.ajic.2010.09.007

Mahfouz, A. A., Al-Zaydani, I. A., Abdelaziz, A. O., El-Gamal, M. N., \& Assiri, A. M. (2014). Changes in hand hygiene compliance after a multimodal intervention among health-care workers from intensive care units in Southwestern Saudi Arabia. Journal of epidemiology and global health, 4(4), 315-321. https://doi.org/10.1016/j.jegh.2014.05.002

Ministry of Local Government, Rural Development and Cooperatives, International Centre for Diarrheal Diseases Research, Bangladesh (icddr, b), \& WaterAid Bangladesh. (2014). Bangladesh National Hygiene Baseline Survey-Preliminary Report. Retrieved from https://washmatters.wateraid.org/publications/bangladeshnational-hygiene-baseline-survey-preliminary-report-2014

Mu, X., Xu, Y., Yang, T., Zhang, J., Wang, C., Liu, W., . . Yang, H. (2016). Improving hand hygiene compliance among healthcare workers: an intervention study in a Hospital in Guizhou Province, China. Brazilian Journal of Infectious Diseases, 20(5), 413-418. https://doi.org/10.1016/j.bjid.2016.04.009

Naikoba, S., \& Hayward, A. (2001). The effectiveness of interventions aimed at increasing handwashing in healthcare workers-a systematic review. Journal of Hospital Infection, 47(3), 173-180. https://doi.org/10.1053/jhin.2000.0882

Neo, J. R. J., Sagha-Zadeh, R., Vielemeyer, O., \& Franklin, E. (2016). Evidence-based practices to increase hand hygiene compliance in health care facilities: an integrated review. American journal of infection control, 44(6), 691-704. https://doi.org/10.1016/j.ajic.2015.11.034

Nteli, C., Galanis, P., Koumpagioti, D., Poursanidis, G., Panagiotopoulou, E., \& Matziou, V. (2014). Assessing the effectiveness of an educational program on compliance with hand hygiene in a pediatric intensive care unit. Advances in Nursing, 2014. https://dx.doi.org/10.1155/2014/704232

Pittet, D., Allegranzi, B., Storr, J., Nejad, S. B., Dziekan, G., Leotsakos, A., \& Donaldson, L. (2008). Infection control as a major World Health Organization priority for developing countries. Journal of Hospital Infection, 68(4), 285-292. https://doi.org/10.1016/j.jhin.2007.12.013

Pittet, D., \& Boyce, J. M. (2001). Hand hygiene and patient care: pursuing the Semmelweis legacy. The Lancet 
Infectious Diseases, 1, 9-20. https://doi.org/10.1016/S1473-3099(09)70295-6

Pittet, D., \& Donaldson, L. (2005). Clean care is safer care: the first global challenge of the WHO World Alliance for Patient Safety. Am J Infect Control, 33(8), 476-479. https://doi.org/10.1016/j.ajic.2005.08.001

Pittet, D., Simon, A., Hugonnet, S., Pessoa-Silva, C. L., Sauvan, V., \& Perneger, T. V. (2004). Hand hygiene among physicians: performance, beliefs, and perceptions. Annals of internal medicine, 141(1), 1-8. https://doi.org/10.7326/0003-4819-141-1-200407060-00008

Randle, J., Arthur, A., \& Vaughan, N. (2010). Twenty-four-hour observational study of hospital hand hygiene compliance. Journal of Hospital Infection, 76(3), 252-255. https://doi.org/10.1016/j.jhin.2010.06.027

Randle, J., Clarke, M., \& Storr, J. (2006). Hand hygiene compliance in healthcare workers. J Hosp Infect, 64(3), 205-209. https://doi.org/10.1016/j.jhin.2006.06.008

Rosenthal, V. D., McCormick, R. D., Guzman, S., Villamayor, C., \& Orellano, P. W. (2003). Effect of education and performance feedback on handwashing: the benefit of administrative support in Argentinean hospitals. American journal of infection control, 31(2), 85-92. Retrieved from https://www.ncbi.nlm.nih.gov/pubmed/12665741

Salama, M. F., Jamal, W. Y., Al Mousa, H., Al-AbdulGhani, K. A., \& Rotimi, V. O. (2013). The effect of hand hygiene compliance on hospital-acquired infections in an ICU setting in a Kuwaiti teaching hospital. Journal of infection and public health, 6(1), 27-34. https://doi.org/10.1016/j.jiph.2012.09.014

Santosaningsih, D., Erikawati, D., Santoso, S., Noorhamdani, N., Ratridewi, I., Candradikusuma, D., . . Voor, A. F. (2017). Intervening with healthcare workers' hand hygiene compliance, knowledge, and perception in a limited-resource hospital in Indonesia: a randomized controlled trial study. Antimicrobial Resistance \& Infection Control, 6(1), 23. https://doi.org/10.1186/s13756-017-0179-y

Sax, H., Uckay, I., Richet, H., Allegranzi, B., \& Pittet, D. (2007). Determinants of good adherence to hand hygiene among healthcare workers who have extensive exposure to hand hygiene campaigns. Infection Control \& Hospital Epidemiology, 28(11), 1267-1274. https://doi.org/10.1086/521663

Schweizer, M. L., Reisinger, H. S., Ohl, M., Formanek, M. B., Blevins, A., Ward, M. A., \& Perencevich, E. N. (2013). Searching for an optimal hand hygiene bundle: a meta-analysis. Clinical infectious diseases, 58(2), 248-259. https://doi.org/10.1093/cid/cit670

Shahida, S. M., Islam, A., Dey, B. R., Islam, F., Venkatesh, K., \& Goodman, A. (2016). Hospital Acquired Infections in Low and Middle Income Countries: Root Cause Analysis and the Development of Infection Control Practices in Bangladesh. Open Journal of Obstetrics and Gynecology, 6(01), 28. https://dx.doi.org/10.4236/ojog.2016.61004

Stout, A., Ritchie, K., \& Macpherson, K. (2007). Clinical effectiveness of alcohol-based products in increasing hand hygiene compliance and reducing infection rates: a systematic review. Journal of Hospital Infection, 66(4), 308-312. https://doi.org/10.1016/j.jhin.2007.04.017

Uneke, C. J., Ndukwe, C. D., Oyibo, P. G., Nwakpu, K. O., Nnabu, R. C., \& Prasopa-Plaizier, N. (2014). Promotion of hand hygiene strengthening initiative in a Nigerian teaching hospital: implication for improved patient safety in low-income health facilities. The Brazilian Journal of Infectious Diseases, 18(1), 21-27. https://doi.org/10.1016/j.bjid.2013.04.006

Vindigni, S. M., Riley, P. L., \& Jhung, M. (2011). Systematic review: handwashing behaviour in low-to middle-income countries: outcome measures and behaviour maintenance. Tropical Medicine \& International Health, 16(4), 466-477. https://doi.org/10.1111/j.1365-3156.2010.02720.x

Whitby, M., McLaws, M. L., \& Ross, M. W. (2006). Why healthcare workers don't wash their hands: a behavioral explanation. Infection Control \& Hospital Epidemiology, 27(5), 484-492. https://doi.org/10.1086/503335

Whitby, M., Pessoa-Silva, C. L., McLaws, M. L., Allegranzi, B., Sax, H., Larson, E., . . . Pittet, D. (2007). Behavioural considerations for hand hygiene practices: the basic building blocks. Journal of Hospital Infection, 65(1), 1-8. https://doi.org/10.1016/j.jhin.2006.09.026

World Health Organization [WHO]. (2009a). A Guide to the Implementation of the WHO Multimodal Hand $\begin{array}{llll}\text { Hygiene Improvement Strategy. } & \text { Retrieved }\end{array}$ http://www.who.int/gpsc/5may/Guide_to_Implementation.pdf

World Health Organization [WHO]. (2009b). WHO guidelines on hand hygiene in health care. Retrieved from 
http://apps.who.int/iris/bitstream/10665/44102/1/9789241597906_eng.pdf

World Health Organization [WHO]. (2011). Report on the burden of endemic health care-associated infection worldwide. Retrieved from http://apps.who.int/iris/bitstream/10665/80135/1/9789241501507_eng.pdf

\section{Copyrights}

Copyright for this article is retained by the author(s), with first publication rights granted to the journal.

This is an open-access article distributed under the terms and conditions of the Creative Commons Attribution license (http://creativecommons.org/licenses/by/4.0/). 